\title{
Myocardial Fat: Physiological or Pathological? A Case Report
}

\author{
Ariyarathna $\mathrm{HTDW}^{*}$ \\ Department of Forensic Medicine, Faculty of Medical Sciences, University of Sri Jayewardenepura. Sri Lanka.
}

\begin{abstract}
The presence of myocardial fat is not a finding that is infrequent during postmortem examinations. The case under discussion showed an extensive amount of fat, markedly on the right and mildly on the left ventricles. The differentiation of physiological fat from pathological entities is essential. In this case, two entities were initially suspected namely Arrhythmogenic Right Ventricular Cardiomyopathy/Dysplasia and excessive physiological fat deposition of the heart. A 66-year-old averagely build, a mentally subnormal, previously healthy female was found dead after a nonspecific, mild, general ill-health. Trauma and the possibility of poisoning were excluded. The cause of death was concluded as coronary artery disease with further investigations. The incidental finding of an excessive amount of fat as seen in this case warrants explanations and future research in Sri Lanka.
\end{abstract}

Keywords: Aging, Arrhythmogenic Right Ventricular Cardiomyopathy/Dysplasia (ARVC/D), physiological myocardial fat

Received: 28 Oct, Revised version accepted: 29 Dec 2020, Published: 31 Dec 2020. *Corresponding author: Ariyarathna HTDW, $\bigotimes$ Email: ariyaratna@sjp.ac.lk (D) https://orcid.org/0000-0002-4266-5508

Cite this article as: Ariyarathna HTDW. Myocardial Fat: Physiological or Pathological? A Case Report. MedicoLegal Journal of Sri Lanka, 2020;8(2):32-35. DOI: http://dx.doi.org/10.4038/mljsl.v8i2.7423

Copyright: @ 2019 with the Medico-legal Journal of Sri Lanka.

This is an open-access article distributed under the terms of the Creative Commons Attribution 4.0 International License, which permits unrestricted use, distribution, and reproduction in any medium provided the original author and source are credited.

\section{Introduction}

Research interests have been increased to study the relationship between the amounts of fatty infiltration of the heart in myocardial diseases. The literature review had shown that a routine autopsy may fail to notice clear distinction among normal and pathological fatty infiltration creating diagnostic difficulties. ${ }^{[1]}$ In this patient both the epicardial fat and fat within the myocardium illustrated a marked increase. Two important aspects were recognized to be excluded before arriving at a diagnosis in this patient. The first, is the exclusion of any pathological fat deposition of her heart and the next is the exclusion of any possible contribution or causation to cause death by that finding. When more than one pathological entities are competing for the cause of death, it is the duty of the forensic pathologist to employ all possible ancillary investigations along with the history and postmortem findings.

The postmortem CT was not facilitated in the hospital concerned and the special staining of Masson's trichrome was performed to reach the correct diagnosis. There had been incidents where pathological fatty infiltration (adipositas cordis) was misdiagnosed as ARVC/D indicating the importance of the correct interpretation of all the investigative findings. ${ }^{[2]}$

\section{Case report}

A 66 year, mentally subnormal, averagely build, unmarried female with no previously diagnosed chronic illnesses had complained of general ill health with the loss of appetite 3 days prior. It was not abnormal to the family members of her odd behaviour as she had on and off chosen to refuse regular meals for few days and then without any intervention or treatment, she used to generally feel better. But on the third day of the present episode, she was found dead one morning. No family history of premature deaths was reported. No external or internal injuries were there suggestive of any trauma contributing to death. No features suggesting heart failure such as ankle oedema were there. The toxicological analysis was also negative for common poisons. No brain, lung, liver, kidney, or other organ pathology was detected both macroscopically and microscopically. The heart was $290 \mathrm{~g}$ in weight and in normal shape. Heart valves and chambers were within normal limits and no features of fresh or old myocardial infarctions were there. The right ventricular wall was epicardially replaced with a macroscopical thick fat layer at the free wall mainly and the width was $7 \mathrm{~mm}$. The myocardium shows apparent fatty tissue even in trabeculae (Fig.1). 

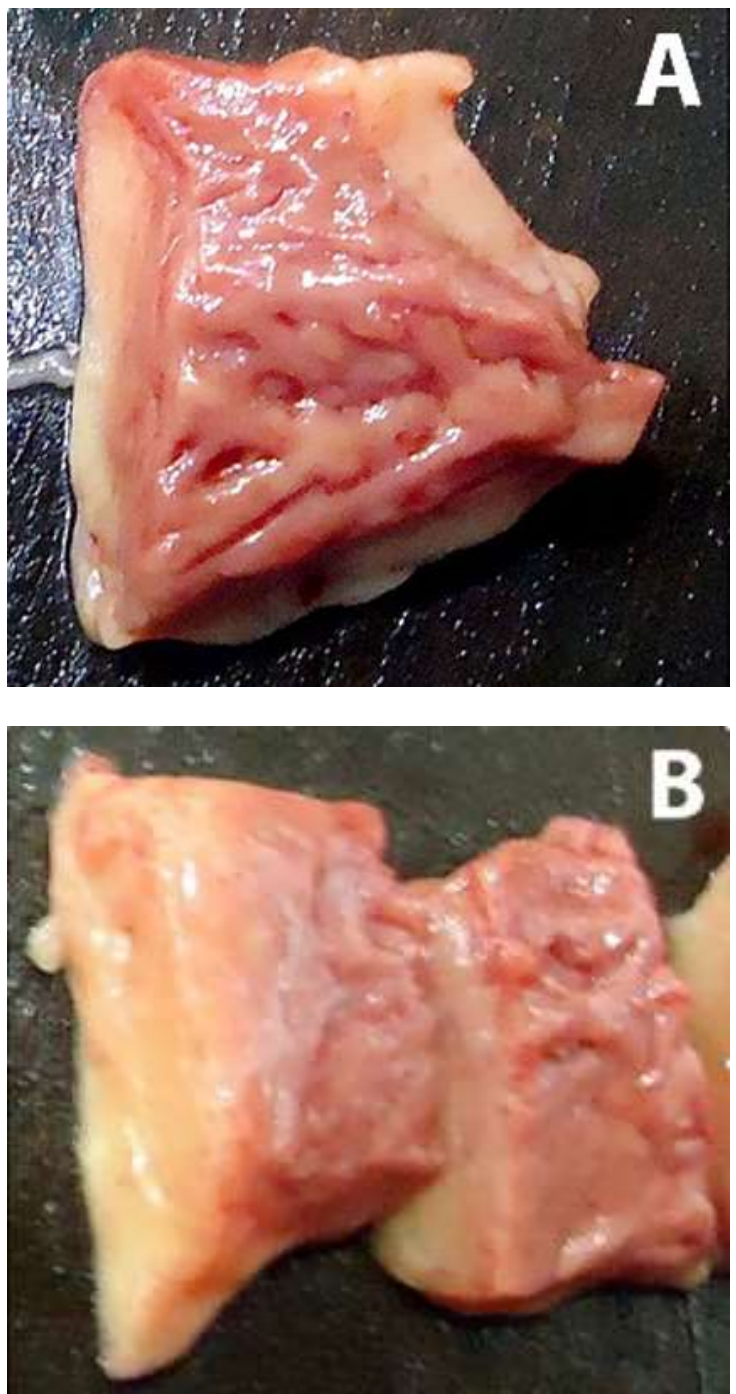

Figure 01. A \& B. Right ventricular free wall with excessive fat infiltration even in trabeculae. The wall thickness was increased (sections obtained for histopathology).

The left ventricular myocardium was visually excessively yellowish-brown (Fig. 2). The anterior descending artery, right coronary artery, and circumflex artery were obstructed with atherosclerosis $90 \%, 70 \%$ and $30 \%$ respectively. The BMI was around 23. No history of past episodes of IHD, diabetes mellitus or hypertension or any other metabolic diseases was noted. She was physically fit excluding muscular dystrophies. The aorta showed complicated atheroma, especially on the abdominal aorta. Circle of Willis and carotid arteries showed mild atheroma formation.
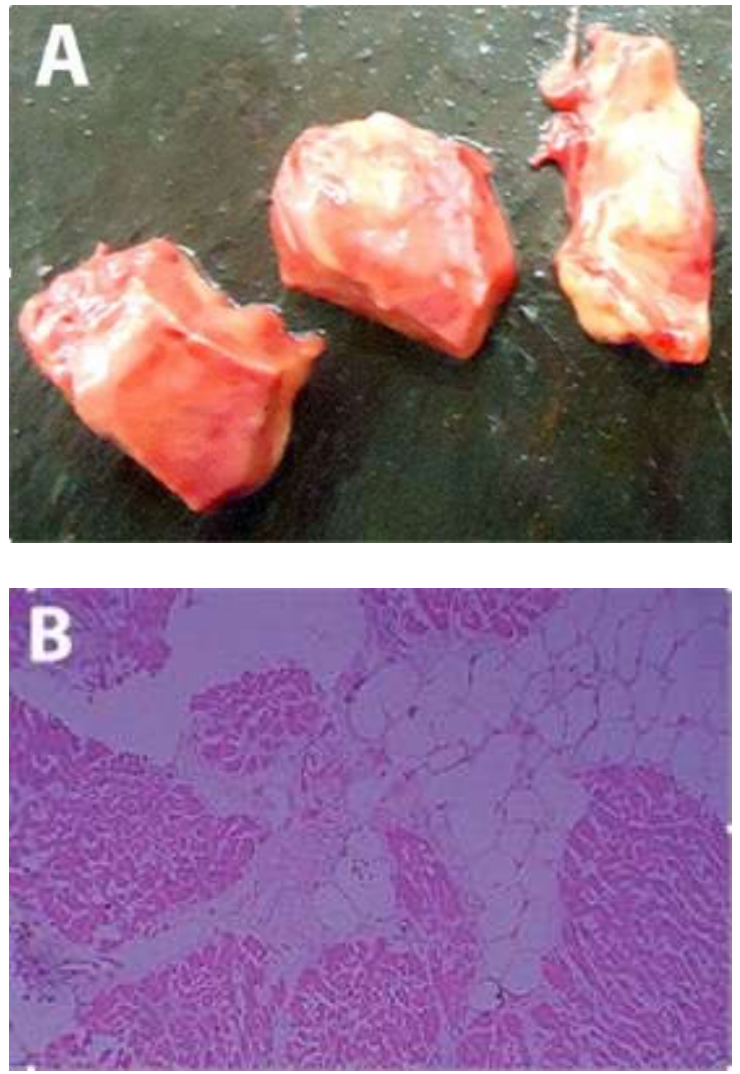

Figure 02. Left ventricular free wall with excessive fat in it. B. H\&E.x10. Fat infiltration into the myocardium of the left ventricle.

The right ventricle microscopically showed fibrofatty replacement with intervening unaffected myocytes with a thick epicardial fat layer. The left ventricle showed fatty infiltration within the myocardium without any inflammatory cells. The Masson's trichrome staining showed increased amounts of fibrous tissues with blue-green. No lipofuscin stains were seen (Fig.3).

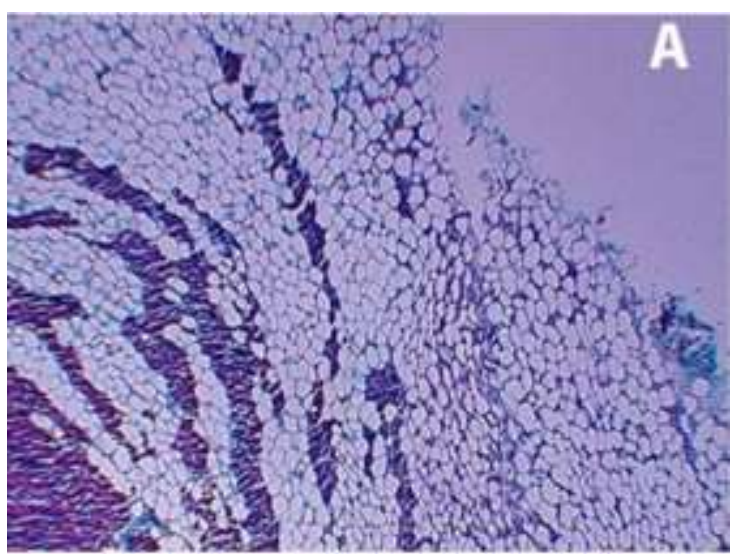




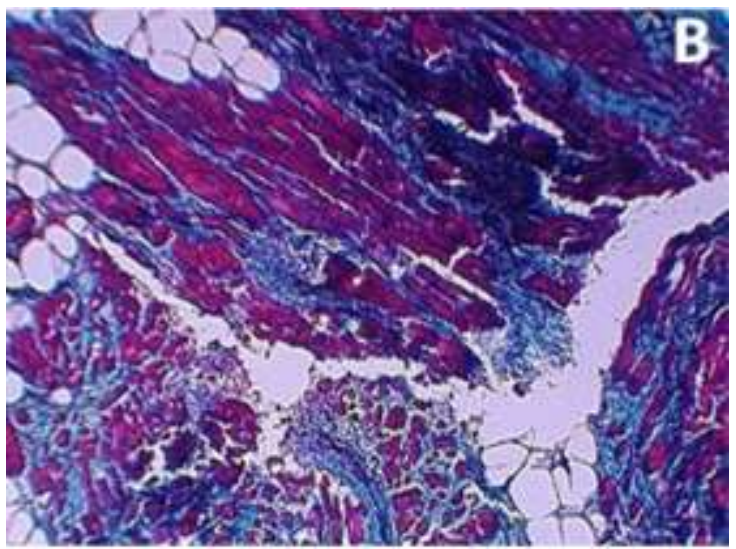

Figure 03. A. Histopathology of the right ventricular free wall with fat infiltration, Masson's trichrome stain $x$ 4. B. Right ventricular free wall with Masson's trichrome stain X 40 to illustrate fibrosis of the myocytes.

Initially, the cause of death was kept under investigation, and with the investigative results, the cause of death was attributed to coronary artery disease ascribing the excessive cardiac fat as physiological or due to aging.

\section{Discussion}

Since cardiac adipose tissues are seen both in physiological and pathological entities, it is essential to understand the characteristic fat patterns in order to differentiate the two. Few conditions that need to be considered in relation to this case, are the nonpathological cardiac fat, Arrhythmogenic Right Ventricular Cardiomyopathy/Dysplasia (ARVC/D), post-myocardial infarction, lipomatous metaplasia, lipomatous hypertrophy of the interatrial septum and other miscellaneous diseases such as muscular dystrophies, lipoma and liposarcomas.

Non-pathological cardiac fat is seen mostly in the right ventricle rather than in the left. In a study, it was revealed that right ventricular adipose tissue was detected in $85 \%$ of patients in those who died due to non-cardiac diseases. ${ }^{[3]}$ Some studies have also shown that adipose tissue was detected in around $50 \%$ of the cases. ${ }^{[4]}$

In minor adipose infiltration in the physiological range, it is only seen around the blood vessels/nerves of the right ventricle. Histological studies have shown that the favourite place for the fat to deposit is on the right ventricle especially the anterolateral and apical walls. A tiny amount of fat had been noticed on the apical left ventricle as well. With heavy fat infiltration, the fat tends to dissociate the muscle bundles rather than to affect the displacement of myocytes. ${ }^{[5]}$ Usually, the fat infiltration is confined to the outer half of the right ventricular wall although in extensive fatty infiltration it can accommodate the full thickness up to the endocardium and even in the trabeculae. The most important point to be highlighted here is the appreciation of the right ventricular thickness. It is speculated that the right ventricular thickness increases with the amount of increase in the fat in the physiological state.

ARVC/D is mostly a familial condition and characterized by fibro-fatty replacement of the ventricular myocardium. It is a progressive disease that affects more commonly males than females. The progression is said to peak in the age range of 15-20 years, the median age is 30 years. $^{[5]}$ The extent of the disease and the electric instability resulted in arrhythmias determine the nature of sudden death. But none of the features are compatible in this case.

Since the fat infiltration of the right ventricle is favoured by both the physiological aging process and pathological entity of ARVC/D, it is crucial to distinguish ARVC/D from the physiological process. In ARVC right ventricle is the place of choice though both ventricles can also be affected with a difference of a certain percentage. The left ventricle is alone affected only rarely. The replacement of the right ventricular wall with attenuation is the gross pathological feature. Such change often results in dilation of the right ventricle. ${ }^{[6]}$ Patchy inflammatory infiltrates along with fibro-fatty tissue either globally or segmentally should also be noted. The degree of thinning of the right ventricle is at times so severe, it is said that the wall is merely translucent with dilatation of the cavity because of the replacement of the muscle fibers with adipose tissue. In the case under discussion the right ventricle is in fact thicker than normal $(0.7 \mathrm{~mm})$ and not dilated. The demographic data is also not compatible with the natural history of the disease. But few features were somewhat compatible with the feature of ARVC/D such as fatty fibro infiltration. Degenerated myocytes and cellular infiltrations are considered quite strong indicators to diagnose ARVC and in this case, such findings were not evident. A study had revealed that the significance of fat infiltration is varied according to the location and fat in the right ventricle and was most commonly to aging followed by postmyocardial infarction and ARVD. ${ }^{[7]}$ The macroscopical features such as the location of the fat, myocardial thickness, and the ventricular size along with microscopical features are helpful to differentiate pathological and physiological cardiac adipocere.

The other differential diagnosis that needs consideration, in this case, is the fatty infiltration after myocardial infarction (post-myocardial cardiomyopathy). Here, no acute or old changes were seen macroscopically although microscopically fibrosis was evident. The presence 
of fat in this entity is mostly subendocardial unless it is a transmural MI. ${ }^{[8]}$ But in this case, the right ventricle was mostly affected epicardially disregarding this possibility. The other differential diagnoses such as cardiac adipose tissue (lipotoxic cardiomyopathy) myotonic dystrophy ${ }^{[9]}$ and associated fatty infiltration, Fabry disease were not compatible in this case. ${ }^{[10]}$

After the postmortem examination, the cause of death was kept under investigation to further study about severe fatty infiltration. Since the ARVC/D was excluded, the excessive fat was ascribed as the physiological/aging process, though the amount seen in this case beyond the physiological amount of fat seen in a comparable deceased. Literature review had emphasized that in certain occasions the routine autopsy is unable to make a clear distinction amongst the normal and abnormal amounts of fatty infiltration leading to diagnostic difficulties.[4] On the other hand, the clinical importance (contribution for death) of physiological fat infiltration may also be inconclusive because there are facts forwarded both for and against in the literature review.[10 \&11]

\section{Conclusions}

Varying amounts of myocardial fat is not that uncommon in autopsy examinations due to physiological and pathological entities. When cardiac fat is excessive and is seen in abnormal places as in this case, the causative or contributory nature of the cause of death should be investigated differentiating the physiological and pathological entities. Thorough knowledge is compulsory to be able to distinguish the physiological and pathological myocardial fat in order not to overdiagnose - especially of ARVC/D either from biopsy, radiologically or in the postmortem examinations.

\section{Acknowledgements}

Dr. Sherin Suzai (Consultant Histopathologist), Colombo South Teaching Hospital for preparation of histology slides, Dr. Dulani Beneragama (Senior Lecturer/Consultant Histopathologist, Department of Pathology) for assisting in reading the histopathology slides and Mr. M.S.E. Premalal (Technical officer) for micro- photographing from the Department of Pathology, Mr. Wasantha Manawadu for the IT assistance (Technical Officer, Dean's office, USJ).

\section{Disclosure statement}

Conflict of interest: The author declares that there is no conflict of interest.

Funding: None

\section{References}

1. Domenico C, Guy F, Frank IM, William JM, Andrea N, Gaetano T, et al. Arrhythmogenic
Right Ventricular Dysplasia/Cardiomyopathy. Circulation. 2000 Mar 21;101(11):e101-6. DOI: http://doi.org/ 10.1161/01.CIR.101.11.e101

2. Marcus F, Basso C, Gear K, Sorrell V. Pitfalls in the Diagnosis of Arrhythmogenic Right Ventricular Cardiomyopathy/Dysplasia. The American Journal of Cardiology. 2010;105(7):1036-39. DOI: 10.1016/j.amjcard. 2009.11.033

3. Tansey DK, Aly Z, Sheppard MN. Fat in the right ventricle of the normal heart. Histopathology. 2005;46(1):98-104. DOI: http://doi.org/ 10.1111/j.1365-2559.2005.02054.X

4. Mu J, Zhang G, Xue D, Xi M, Qi J, Dong H. Sudden cardiac death owing to arrhythmogenic right ventricular cardiomyopathy: Two case reports and systematic literature review.

5. de la Grandmaison GL, Le Bihan C, Durigon M. Assessment of right ventricular lipomatosis by histomorphometry in control adult autopsy cases. Int J Legal Med. 2001 Oct 1;115(2):105-8. DOI: https://doi.org/10.1007/s004140100224

6. Arrhythmogenic Right Ventricular Dysplasia/Cardiomyopathy (ARVD/C) Thoracic Pathology: A Volume in the High Yield Pathology Series (Expert Consult - Online and Print) 1st Edition [cited 2020 Oct 17]. Available from: https://doctorlib.info/medical/thoracicpathology/254.html

7. Jacobi AH, Gohari A, Zalta B, Stein MW, Haramati LB. Ventricular myocardial fat: CT findings and clinical correlates. $\mathrm{J}$ Thorac Imaging. 2007 May 1;22(2):130-5. DOI: http://doi.org/ 10.1097/01.rti.0000213576.39774.68

8. Selthofer-Relatić K, Belovari T, Bijelić N, Kibel A, Rajc J. Presence of Intramyocardial Fat Tissue in the Right Atrium and Right Ventricle Postmortem Human Analysis. Acta Clin Croat. 2018 Mar;57(1): 122-9. DOI: 10.20471/acc.2018.57.01.15

9. Samanta R, Pouliopoulos J, Thiagalingam A, Kovoor P. Role of adipose tissue in the pathogenesis of cardiac arrhythmias. Heart Rhythm. 2016 Jan 1;13(1):311-20. DOI: 10.1016/j.hrthm.2015.08.016

10. Bertaso AG, Bertol D, Duncan BB, Foppa M. Epicardial Fat: Definition, Measurements and Systematic Review of Main Outcomes. Arq Bras Cardiol. 2013 Jul;101(1):e18-28. DOI: 10.5935/abc. 20130138

11. Anumonwo JMB, Herron T. Fatty Infiltration of the Myocardium and Arrhythmogenesis: Potential Cellular and Molecular Mechanisms. Front Physiol. 2018 [cited 2020 Oct 17]. Available from: https://www.frontiersin.org/ articles/10.3389/fphys.2018.00002/full 